\title{
An end-user evaluation to explore the effectiveness of cartograms for mapping spatial accessibility
}

\author{
Aldo Arranz-López ${ }^{\mathrm{a}, *}$, Julio A. Soria-Lara ${ }^{\mathrm{b}}$, Amor Ariza-Álvarez ${ }^{\mathrm{b}}$ \\ ${ }^{a}$ Institute für Humangeographie, Goethe Universität, Frankfurt am Main, Germany.arranz-lopez@geo.uni-frankfurt.de \\ ${ }^{b}$ Transport Research Centre -TRANSyT-, Universidad Politécnica de Madrid, Spain. julio.soria-lara@upm.es \\ * Corresponding author
}

Keywords: Practitioners, maps, active modes, cartography

\begin{abstract}
:
In the era of modern computer graphics and advanced visualization techniques, it seems more relevant than ever to design maps that support decision-making and present planning outcomes in an easily accessible format to practitioners and decision-makers alike. A case in point is the need for effective maps that support the development of policies that enhance non-motorized relative accessibility to major activities. Relative accessibility is defined as the proportion of opportunities available on foot/bicycle to an individual with defined characteristics at a selected location, relative to an individual from a reference group at that location. However, traditional methods for mapping accessibility (e.g., choropleth and grid-based maps) have limitations to highlight spatial dissimilarities of non-motorized accessibility for different socio-economic groups. Cartograms, a type of map that presents geographic space as distorted based on quantitative information, are seen as part of the solution. Under this context, this study aims to evaluate the practitioners' perspective on the effectiveness of grid-based maps and cartograms (diffusion-based and Dorling) (Figure 1) for mapping relative non-motorized accessibility to retail, taking Zaragoza (Spain) as a case study.
\end{abstract}
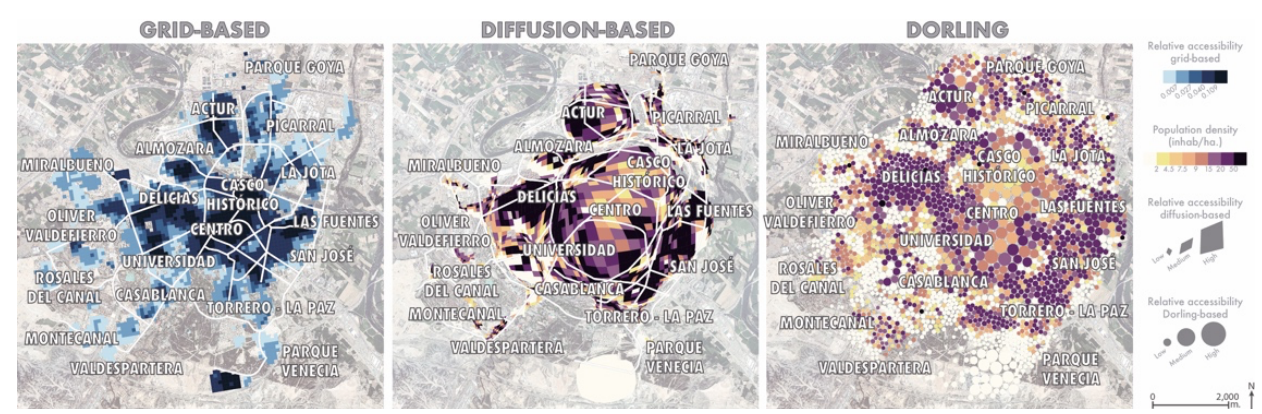

Figure 1. Example of grid-based maps (left), diffusion-based cartograms (middle), and Dorling cartograms (right)

The evaluation of the cartograms' effectiveness was based on 30 semi-structured interviews from local practitioners working in the transport sector, including rated statements based on a 5-point Likert scale for evaluating four cartographic aspects: (i) design; (ii) readability, (iii) suitability, and (iv) usability. Additionally, an open-ended question was added where participants provided a more detailed viewpoint (when necessary) about the aforementioned aspects. Before the semi-structured interview, the research team provided each participant with rules and instructions to ensure uniform application of the research tool, including the types of cartograms, their characteristics, and their process of design.

The results for the "cartographic design" (Figure 2a) showed that 55\% agreed that the design of the grid-based maps was appropriate to showcase relative non-motorized accessibility; however, only $9 \%$ held this opinion for Dorling cartograms. Diffusion-based cartograms fared a bit better as $18 \%$ expressed a positive view. The use of additional layers (e.g., roads and streets) seems like an important explanation of the preference for diffusion-based over Dorling cartograms. Practitioners frequently indicated during open questions that "the design of Dorling cartograms would strongly improve if the main roads and streets layers would have been included in the final layout. On the contrary, the spatial context is not well-described, originating misinterpretations regarding city locations and accessibility values." However, Dorling cartograms have the inherent technical limitation that they cannot include additional spatial information, as their cartographic outputs (mainly circles with the distorted variable) are not spatially continuous. Regarding the effect of pallet color on cartographic design, practitioners considered the colors used as appropriate, while several of them signaled that they "would have opted for less population density intervals, making easier their visualization." For "readability" (Figure $2 \mathrm{~b})$, almost two-thirds (62.5\%) of participants agreed that the readability of grid-based maps was appropriate to distinguish places with the highest and lowest values of non-motorized accessibility for each population cluster. This percentage decreased to less than one-third (31.25\%) for diffusion-based and to $13.3 \%$ for Dorling cartograms. 
Nevertheless, it is worth to mentioning that a majority of practitioners $(60 \%)$ commented that diffusion-based cartograms were particularly appropriate for identifying spatial transitions from the highest to lowest values of non-motorized accessibility. The difficulty to read two variables simultaneously (accessibility and population density) was another weakness of cartograms discussed during the interviews. More than half (56.66\%) felt that both types of cartograms would be more readable if they "only showed one variable rather than two." Despite the interviewer's effort to systematically emphasize that presenting two variables in the same cartographic layout (accessibility and population density) can provide practitioners with more information than grid-based maps, respondents continued underlying during open questions that "they usually mapped accessibility as a single variable in daily practice, which facilitated the understanding of maps specially during interactions with non-expert people."

Design (a)

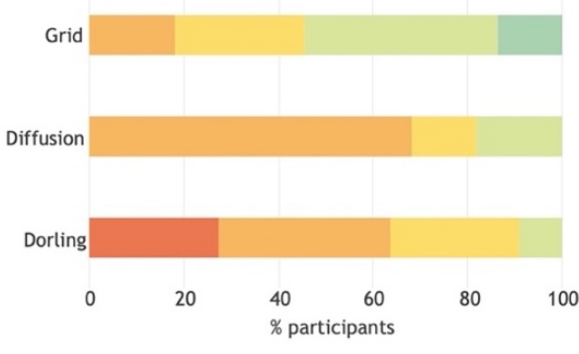

Suitability (c)

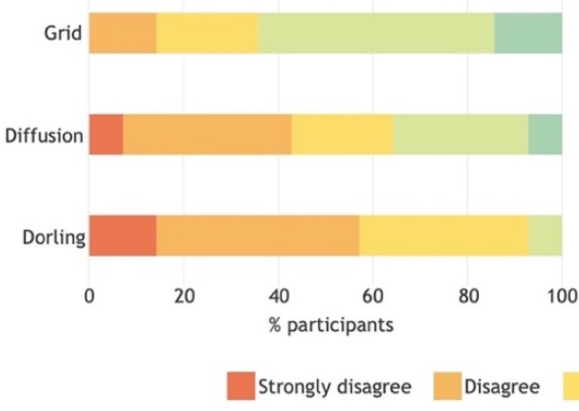

Readability (b)

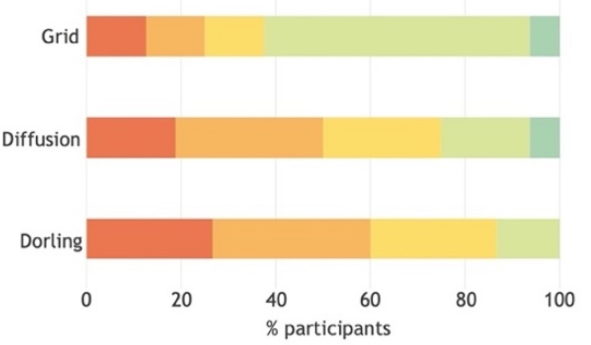

Usability (d)

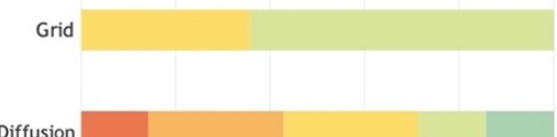

Dorling

100

Neutral Agree Strongly agree

Figure 2. Cartographic aspects included in the end-user evaluation

Regarding the "suitability" (Figure 2c) of grid-based maps and cartograms to show spatial dissimilarity patterns of relative non-motorized accessibility between population groups, $64 \%$ of participants agreed that grid-based maps were suitable. Diffusion-based cartograms were deemed appropriate by $35.71 \%$, while only a few practitioners (7.14\%) found Dorling to be suitable. As emphasized during the open question, "the problem can be related to the circles used to distort the accessibility levels, since this circular shape would limit the capacity to detect spatial dissimilarities when such spatial dissimilarities are not strongly present." However, this spatial distinction seemed easier to achieve by using diffusionbased cartograms even when these spatial dissimilarities patterns are less in evidenced (as spatial outliers) in a given context. A majority of practitioners declared that "the most effective way to visually identify spatial dissimilarity patterns of relative non-motorized accessibility was based on combining grid-based maps and diffusion-based cartograms." The last part of the interviews focused the "usability" of grid-based maps and cartograms in daily practice (Figure 2d). Almost two-thirds of practitioners (64.28\%) agreed that grid-based maps were a usable visualization technique for representing relative non-motorized accessibility. The percentage for diffusion-based cartograms was $30 \%$, falling to $20 \%$ for Dorling. During the open question, almost one-third of respondents $(30 \%)$ declared that "it is difficult to change a strongestablished tradition in visualization techniques, as a transition to other techniques would require additional training for employees among others." It was also mentioned by $18 \%$ of practitioners that "consultancy offers are frequently constrained to conducting specific spatial analysis according to the call for tender, which usually limits the capacity to add new visualization techniques."

The practitioners' inexperience concerning cartograms could have originated a lower interest in perceiving their added value for visualizing relative non-motorized accessibility. It is also noted that cartograms are used at global and regional scales where different spatial patterns can be more easily visualized. However, this study tried to depict comparisons in a smaller and continuous geographic area. Further research should address additional end-user experiments, using a wide range of cartograms (e.g., value-by-alpha, rectangular) paying special attention to design issues that make them more attractive and useful in the field of transport planning. 